Research Article

\title{
Use of the Human Walking Gait Cycle for Assistive Torque Generation for the Hip Joint Exoskeleton
}

\author{
Riska Analia (D), Jan Hong, Joshua Mangkey, Susanto, Daniel Pamungkas $(\mathbb{D}$, \\ Hendawan Soebhakti, and Abdullah Sani
}

Department of Electrical Engineering, Politeknik Negeri Batam, Batam, Indonesia

Correspondence should be addressed to Daniel Pamungkas; daniel@polibatam.ac.id

Received 12 January 2021; Revised 26 July 2021; Accepted 12 November 2021; Published 7 December 2021

Academic Editor: Changsheng Li

Copyright ( 2021 Riska Analia et al. This is an open access article distributed under the Creative Commons Attribution License, which permits unrestricted use, distribution, and reproduction in any medium, provided the original work is properly cited.

\begin{abstract}
The development of an assistive robot to assist human beings in walking normally is a difficult task. One of the main challenges lies in understanding the intention to walk, as an initial phase before walking commences. In this work, we classify the human gait cycle based on data from an inertial moment unit sensor and information on the angle of the hip joint and use the results as initial signals to produce a suitable assistive torque for a lower limb exoskeleton. A neural network module is used as a prediction module to identify the intention to walk based on the gait cycle. A decision tree method is implemented in our system to generate the assistive torque, and a prediction of the human gait cycle is used as a reference signal. Real-time experiments are carried out to verify the performance of the proposed method, which can differentiate between various types of walking. The results show that the proposed method is able to predict the intention to walk as an initial phase and is also able to provide an assistive torque based on the information predicted for this phase.
\end{abstract}

\section{Introduction}

Research into wearable assistive robots has recently become popular, and one example of such a robot is the exoskeleton. Most researchers have focused on developing wearable robots for the lower limbs, which can be used in real-time applications for both military and medical purposes, such as assistive robots for people who have lost their ability to walk. A decrease in the ability to walk can be caused by many factors, such as aging, complications from a stroke, or a spinal cord injury. A wearable exoskeleton can therefore be useful in terms of enhancing the ability to walk. The most challenging aspect, which should be emphasized when developing an exoskeleton, is to make it as comfortable as possible for users to wear on their body.

An overview of the various types of exoskeleton was presented in [1], including the design of lower limb exoskeletons, the sensors and actuators that are used, and other relevant aspects. Compared with the other joints, the human hip requires the most effort from an assistive robot [2]. A hip exoskeleton can create positive outcomes in terms of assisting people to walk and can also be used as a rehabilitation device, as it can provide torque to the human hip $[3,4]$. The authors of $[5,6]$ described the development of the exoskeleton and its use in assisting soldiers in walking. The robot includes several sensors that are used to detect the intention to walk. The combination of a mechanical system and a closed-loop control system allows the robot to avoid unstable situations. The control system reads the forces and torque related to the user's intention without the need to acquire the data of a force, position, or electromyogram signal from the user. The development of ReWalk and ReStore [7] expanded the exoskeleton for use in spinal cord injuries and provided the powered hip and knee motion necessary for walking, standing upright, and climbing and descending stairs.

In the rehabilitation of lower limb disability due to stroke and training in the poststroke period, both dorsiflexion and plantarflexion assistance are provided to facilitate functional gait training. An exoskeleton for spinal cord injuries was introduced by HAL; this robot utilized a gait intention estimator to detect not only the intention related to the start of 
the leg swing, based on the behavior of the center of ground reaction force, but also calculated the swing speed depending on the walking velocity [8]. Assistive torque generation can be produced by using the center of pressure $(\mathrm{CoP})$ to detect the pressure from the user's feet at the start of walking. To improve the balance of walking, a fuzzy control system was used to estimate the state of balance of the user. The assistive torque generated by a motor mounted on the lower limb exoskeleton included supporting torque, gravity compensation torque, and correction torque [9]. An assistive torque device based on human motion prediction was introduced in [10], and this used a predictive artificial neural network (pANN). The human walking gait cycle and the CoP information were used as input signals to the system in order to generate assistive torque based on the user's intention to move.

A novel method of human effort estimation for a knee joint of an exoskeleton was presented in [8,9], and this approach relied on online estimation. Information about the upper body, such as the pose, mass, and center of mass, was not needed; instead, information from the force sensor inside the shoes and the inertial moment unit (IMU) sensor on the shanks of the device was used. To estimate the knee torque required, an equilibrium condition was imposed on the user's shanks. An inverse static analysis of the user's lower limbs was then carried out in order to estimate the torque at each leg joint and to enable real-time implementation.

To provide assistive torque for a lower limb exoskeleton, motion analysis software called Kinovea can be used to measure the parameters of motion [10]. This measures the sitting-to-stance and the back-to-sit phases of the motion parameters. The Lagrangian formulation was used to estimate the assistive torque during phase studies and generated a parabolic blend trajectory in online simulation.

The nonlinear model predictive control method can also be used to provide an assistive strategy for a completely actuated exoskeleton. The authors of [11] introduced a model of a human exoskeleton system that was developed based on multiple simulations. An alternative flexible joint control method for a hip assistive exoskeleton was proposed in [12], which directly controlled the torque to reduce the intrinsic mechanical impedance. This method used a gait synchronizer to estimate the intention to walk based on the angular velocity of the hip. This gait synchronizer was used to generate, recognize, synchronize, and segment the actual walking gait, which provided a method of indirectly perceiving intention.

Most researchers in this area have focused on the walking motion when developing an exoskeleton, in order to ensure that a suitable assistive torque is provided. Prediction of walking intention can ensure that the exoskeleton is as comfortable to use as possible and can provide assistive torque. One of the sensors that can be used in a human exoskeleton to predict motion intention is the IMU. In [13], the human gait cycle was recognized based on IMU information using the decision tree method. In this work, three different gait phases of the passive lower limb exoskeleton were recognized: stance, swing, and push. In $[14,15]$, an
IMU was used to calculate the angle produced by the upper and lower arm during movement. An IMU sensor was also used in a lower limb exoskeleton to detect the angles of the ankle and knee joints, and a force sensor was placed in the insole of the shoe. This mechanism was designed to control the ankle and knee joints of a lower limb exoskeleton to assist children with cerebral palsy [16].

In order to generate a suitable assistive torque, the walking intention of the user needs to be considered, and to achieve this, information on the gait cycle can be used in the detection of motion. This can be done by using multiple sensors to predict the initial walking intention and the gait; for example, Thongsook et al. [13] utilized two IMU sensors and pressure sensors on each leg. In the present study, however, information on the gait cycle is generated based on data from an IMU sensor placed on the chest of the user and information on the angle of the hip joint. A neural network $(\mathrm{NN})$ module is used to perceive the intention to walk based on the gait and to separate it into several frames. The initial walking prediction is then used as input information in order to generate assistive torque using the decision tree method.

\section{Research Method}

In order to demonstrate the potential of the proposed system, a prototype of our lower limb exoskeleton, the human walking gait cycle, and the proposed method are explained in detail in this section.

2.1. Exoskeleton. The exoskeleton developed in this work was constructed based on the mechanical design shown in Figure 1(a). It can be seen from the figure that the design consists of several parts: a PC and battery dock, slide frames, a hip joint, represented as a motor driver and Maxon DC motors, and the layer frames as a mechanical support from the thigh to knee. The support frame between the thigh and the knee joint does not actively move and follows the natural movement of the thigh, since no actuators are mounted on the frame. The PC central controller and the battery are located in the dock; however, in this study, an ordinary PC was used, meaning that our prototype was limited in its movement by the length of the connector. The prototype for this robot was $3 \mathrm{D}$ printed using PLA material. A Maxon 408057 motor was chosen as the actuator for the hip joint of the robot. The robot was also constructed with no conversion gear, so that the maximum torque produced by the actuator was only about $0.227 \mathrm{Nm}$ (based on information from the datasheet). The prototype of this robot can be seen in Figure 1(b), which shows all of the components mounted on the robot and its placement on the user's body. The IMU sensor is located on the chest of the user, while the encoder sensors are placed on the Maxon motors on each hip joint of the robot.

An electrical diagram of the system is shown in Figure 2. It can be seen that the electrics are separated into three blocks: the sensor, the central controller, and the motors/actuators. The sensor block contains an IMU sensor to 


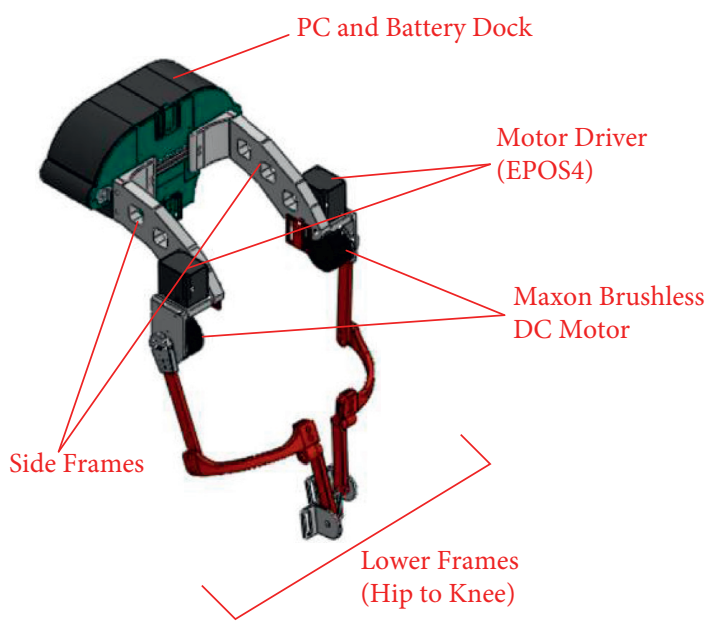

(a)

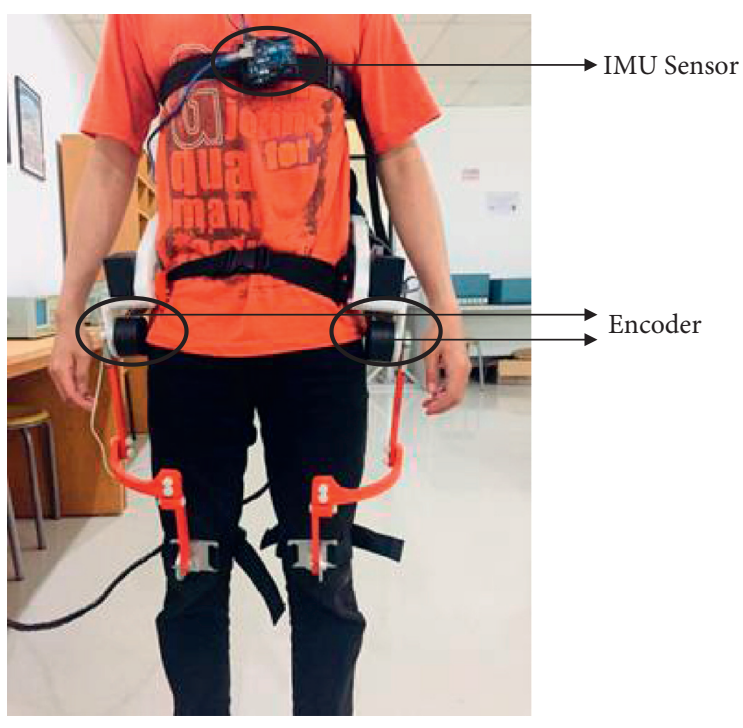

(b)

Figure 1: The proposed exoskeleton. (a) Mechanical design. (b) Photograph of the prototype.

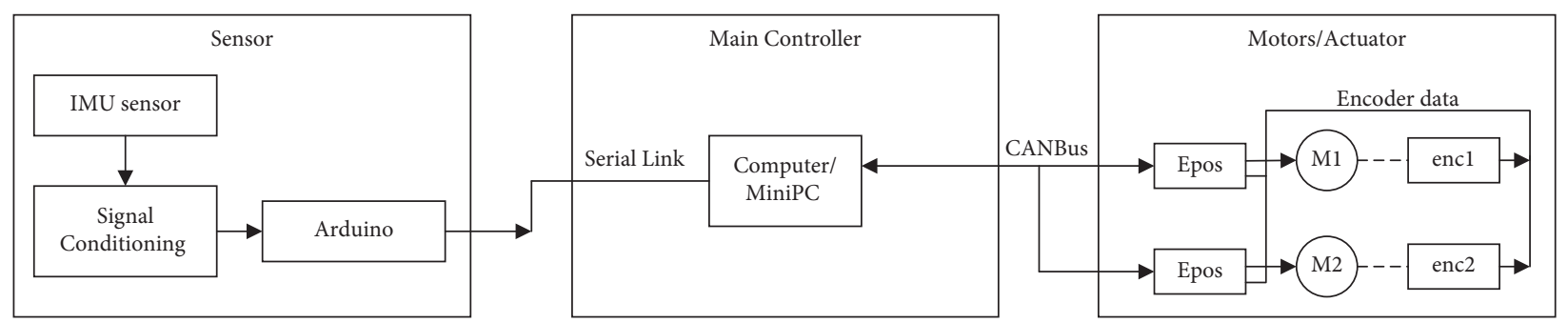

FIgURE 2: Electrical block diagram for the exoskeleton.

capture the changing slope of the chest while walking. The raw data from the IMU are then passed to a signal conditioner to allow these data to be read as required. The output from the signal conditioner is passed to the Arduino. The IMU signal is read from the Arduino, and the data are sent to the main controller via the serial link. The other sensor needed in this system was an encoder. The encoder data represent a transformation of the current signal from the movement of the hip joint into an angle. The angle obtained from the IMU and encoder is used by the main controller as a reference signal for analyzing the intention to walk. In order to generate a suitable assistive torque, the main controller sends the calculated torque to the actuator using the controller area network bus (CAN-Bus) protocol. The sampling frequency for the sensors and controller we set as $100 \mathrm{~Hz}$.

2.2. Classification of the Gait Cycle. The human gait cycle was introduced in [17], and it was shown that each phase of walking is unique, with different leg and hand movements. When the initial movement is identical to the final movement, this is called the gait cycle. Human gait depends on a complex interplay between the major parts of the nervous, musculoskeletal, and cardiorespiratory systems, which are influenced by age, personality, mood, and sociocultural factors. The gait cycle is separated into two periods, namely, the stance and swing phases. In the stance phase, the foot is stepping onto the floor so that it can support the body, whereas the swing phase takes place when the foot is swinging. In the stance and swing phases, several events can be identified, such as the loading response (LR), midstance (MSt), terminal stance (TSt), preswing (PSw), toe off (TO), midswing (MSw), terminal swing (TSw), and heel strike (HS). An illustration of the normal human gait cycle can be seen in Figure 3. Normal human walking motion starts with the TO phase, when a person intends to walk. There is then a transition from the TO phase to the MSw phase, where the angle of the footstep becomes wider than before. The TSw phase indicates that the motion of the swing phase is about to stop, and the rate of change of the angle of the footstep is smaller than before. At the end of the swing phase, HS occurs, which forms the transition to the stance phase. The stance phase begins with an LR, in which the user starts to exert a load on the floor from their body. The user then enters the MSt stage, where the foot exerts the full load of the body of the floor, and the TSt phase represents the end of the walking cycle.

In this work, the phases of walking signal are classified using frames, which are identified from the normal walking gait cycle. These frames are obtained based on the walking pose and the tilt angle of the user's body, and an IMU sensor 


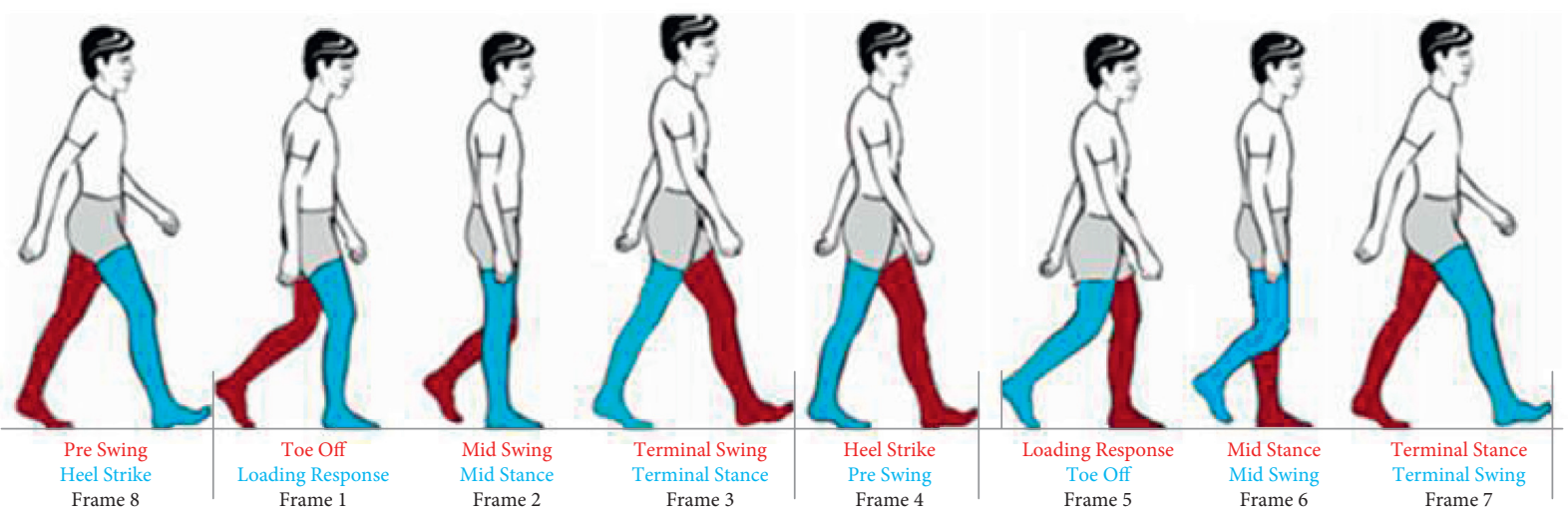

FIgURe 3: The norm output of the gait cycle frame at human walking gait cycle.

is used to detect the angle of tilt as the user walks. The frames used in this work are separated into the eight phases shown in Table 1. A combination of the swing and stance phases for each foot and the tilt angle of the body are used to determine the frame, which forms the output signal. The "+" and "-" signs in Table 1 represent the tilt angle of the body as the user is walking and refer to the left and right sides of the body, respectively. This parameter depends on the initial step taken when walking (i.e., with either the left or right foot). The gait cycle shown in Figure 3 in red refers to the left foot, and the cycle in blue represents the right foot. The first frame shows the left foot in the TO phase and the right foot in the LR phase, while the second frame shows the left foot in the MSw phase and the right in the MSt phase. The third to the eighth frames are then determined as shown in Figure 3. When the user stands still or stops walking, the proposed method will identify this as frame zero.

The classification architecture used in our system is illustrated in Figure 4. In this figure, the left and right foot signals are based on the encoder signal from the motors. Before applying the pretraining process, the IMU sensor and encoder signal need to be normalized to the range zero to one, in order to simplify the learning process for the pretrained NN module. The architecture of the NN consists of three input layers (the IMU sensor and two encoder signals), 10 nodes in the first hidden layer, and 12 nodes in the second hidden layer. The normal walking gait cycle is used in the learning process, as shown in Figure 5, and the pretraining data on the walking gait cycle are classified based on the differences in the position and angle of the foot while the user was walking on a level floor. For example, in Figure 5, when the angle of the left foot is larger than that for the right foot, the network will learn that the left foot is in the midstance position and the right foot in the midswing position, and so on. The resulting signal is divided into eight frames, which are used as training data, in order to classify the walking gait for the left and right feet. The output is a three-digit walking phase, as shown in Table 2, which can be used to determine the intention to begin walking. Data are divided into two parts, namely, training and test data sets. The ratio between two data is $70 \%$ data goes to training set and the rest to the test set. The training process was carried out for around 45,765 iterations to achieve a target error of
0.001 , where the 0.001 was the average loss error generated from the difference between input and output. The results can be seen in Figure 5 for forward and reverse movement.

2.3. Generation of Assistive Torque. The walking gait classification at Section 2.2 will provide the output NN, which is related to the walking gait frame shown in Figure 3. After determining the initial intention to walk, a method needs to be developed to generate torque in order to allow the exoskeleton to provide a suitable level of assistive torque to the user. The assistive torque given for each foot will be generated based on the walking gait cycle frame, sequentially depending on the walking behavior. In order to generate the assistive torque, in this work, the decision tree algorithm has been used, and this was implemented in real time. This algorithm is popular because it is easy to analyze. Moreover, decision tree is effective in predicting an outcome [18]. Each walking frame identified in the previous step was used as a reference for generating the torque. As can be seen from Figures 6 and 7, a two-torque generation scheme is used for the left and right feet. The system detects the change in the angle reported by the IMU before generating the torque. When the IMU increases every 0.1 degrees then it notices that the user is still walking and the exoskeleton will provide assistive torque; when there is no change in the slope of 0.1 from the IMU sensor, then the system detects that the user has stopped walking or is in the started position. The torque is estimated based on the simulation results in [18], in which suitable values are given for the torque in a hip joint exoskeleton. However, our approach is implemented in a real-time application, meaning that the torque reported in [19] must be normalized based on the maximum torque produced by the Maxon motor. The estimated torque is shown in Figures 6 and 7 and is generated by the current produced by the motor as the user is walking. The minus (-) sign for the torque in Figure 6 indicates that the motors are operating in opposite directions.

\section{Results and Discussion}

This section presents the results of our proposed method of identifying intention to walk based on prediction of the human gait cycle. The intention to walk is used in our 
TABLE 1: Classification.

\begin{tabular}{lccc}
\hline Frame & Left foot & Right foot & Tilt angle of the body \\
\hline 1 & Toe off (TO) & Loading response (LR) & - \\
2 & Midswing (MSw) & Midstance (MSt) & - \\
3 & Terminal swing (TSw) & Terminal stance (TSt) & - \\
4 & Heel strike (HS) & Preswing (PS) & - \\
5 & Loading response (LR) & Toe off (TO) & + \\
6 & Midstance (MSt) & Midswing (MSw) & + \\
7 & Terminal stance (TSt) & Terminal swing (TSw) & + \\
\hline
\end{tabular}

Input Layer $\quad 1^{\text {st }}$ Hidden Layer $\quad 2^{\text {nd }}$ Hidden Layer $\quad$ Output Layer

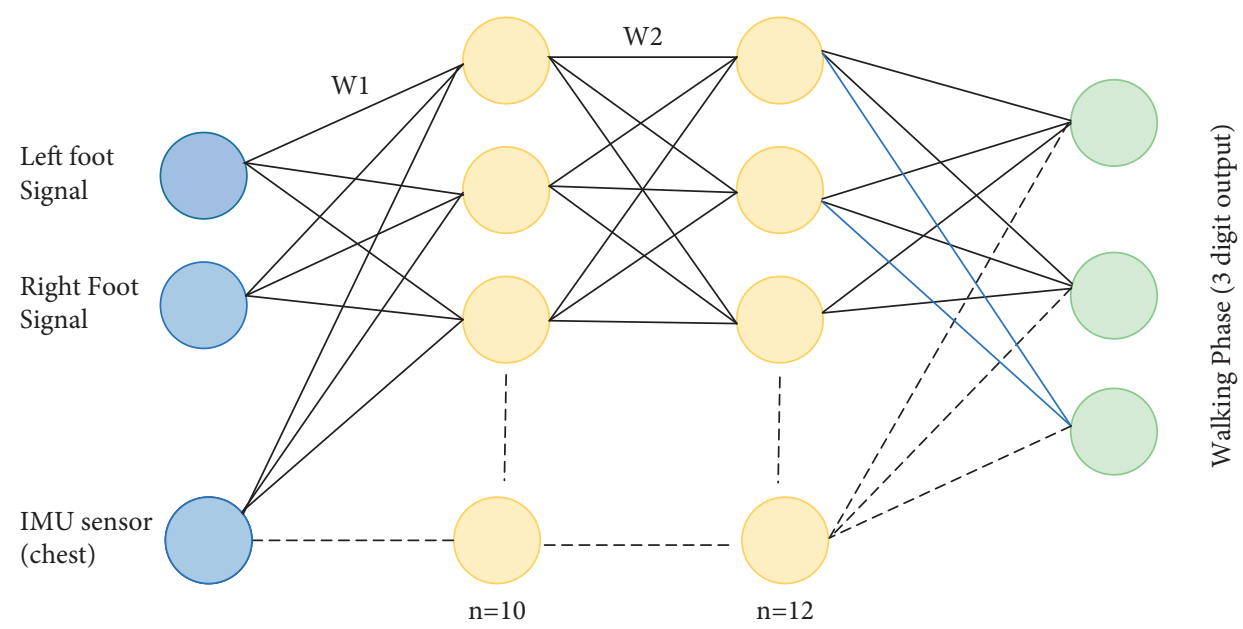

FIgURE 4: Classification architecture of the proposed neural network.

scheme as the initial condition for providing assistive torque. The results for walking intention can be seen in Figure 8 and were collected for a healthy user with height of $170 \mathrm{~cm}$. As can be seen from Figures 8(a) and 8(b), the proposed method was able to identify walking intention based on the gait cycle. The walking intention was detected during walking and ending the walking phase; as seen in Figure 8(a), the walking intention for the left foot detected the toe off phase while loading response for the opposite foot. Meanwhile, when the user stopped walking as seen in Figure 8(b), both legs' intention lied in the stop phase. To enable the reader to better understand the proposed method, Table 3 presents the full results, including the gait phase at each stage, as the user is walking. Table 3 also shows the angles detected for the left and right feet, and the chest angle, which is used as a reference signal for classification of the gait cycle. The complete walking signal can be seen in Figure 9. The data making up this signal are drawn from the encoder on the hip and the IMU signal. At the outset, the IMU signal (marked as "Chest") was mounted on the top between the left and right hips. When user signals the intention to walk by tilting the body to the right, the IMU signal decreases and the system predicts that the user will move the left foot. It is indicated that the signal position at the bottom of all the signals reached 0.3 degrees. This also happens when the body tilts to the left. The signal representing a rightward tilt will then decrease to around 0.2 degrees, indicating that the user has moved the right foot. This cycle will continue until the user stops walking.

When the user's intention to walk has been identified, the next step is to implement the decision tree method in order to allow the exoskeleton to generate the assistive torque. Figure 10 shows the assistive torque produced for a normal walking process on a level floor. Since a conversion gear was not mounted in our mechanical design, the exoskeleton provided the maximum torque, which was $0.227 \mathrm{Nm}$. This limitation meant that a flat signal was provided when the user needed more torque than the motor could supply. This effect also occurred when the user widened their swing step, as shown in Figure 11, or increased their walking pace, as shown in Figure 12. Larger steps taken by the user will cause a flat signal in several of time. However, despite this flat signal, the exoskeleton was able to generate torque in a proper way based on the walking intention signal. It can be seen that the assistive torque follows the change in the slope of the hip joint.

The assistive torque generated in this work was applied only to the hip joint, with a maximum torque of around $0.227 \mathrm{Nm}$, and our prototype was designed with no conversion gear for the left and right feet. The subject felt that this equipment helped him to move his hips, even though the force generated by the exoskeleton was not 


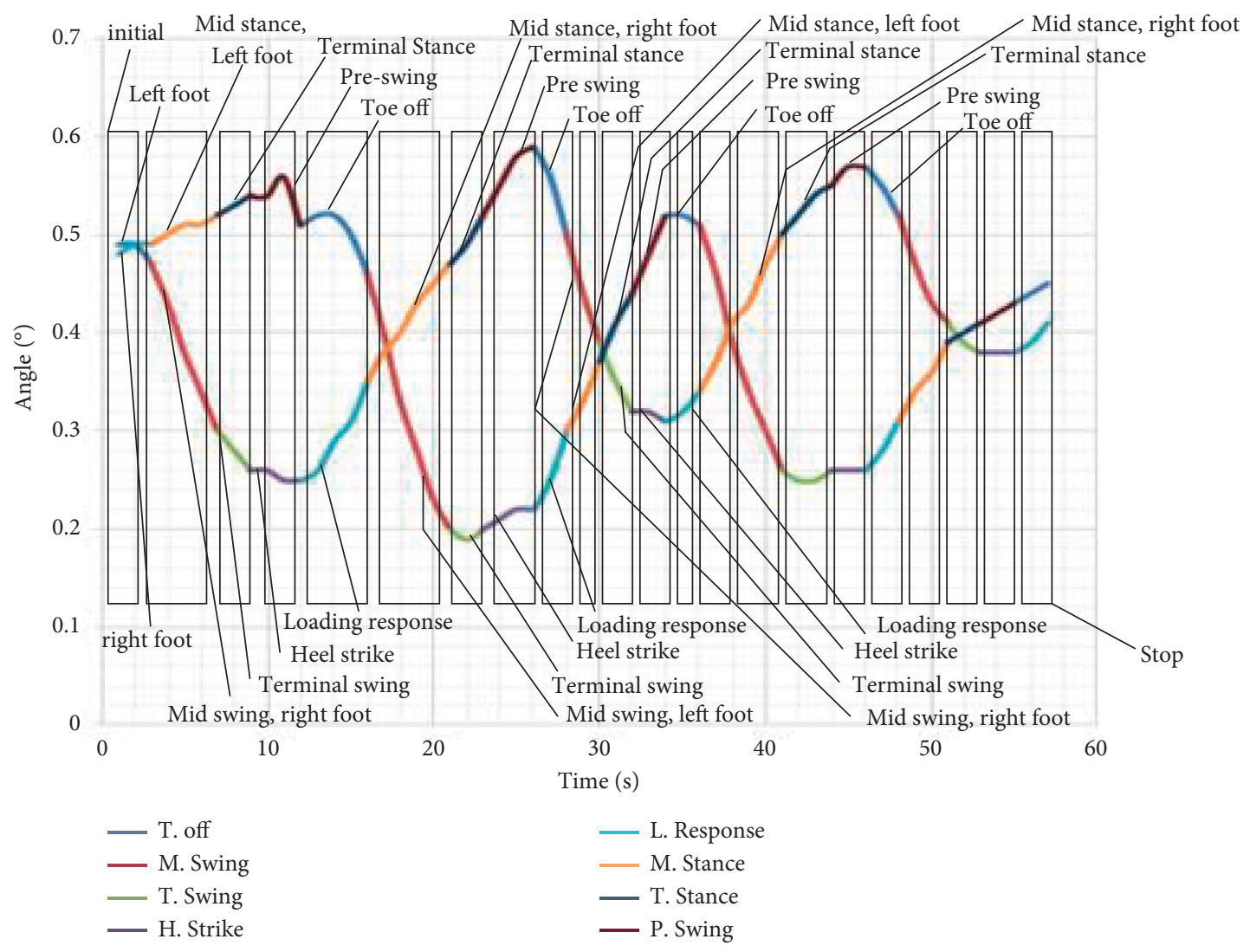

FIGURE 5: Walking gait cycle used in the learning process.

TABLE 2: Output of the NN frames.

\begin{tabular}{lccc}
\hline No. & Output NN & Gait cycle position \\
\hline 1 & 000 & Light leg & Left right \\
2 & 001 & Toe off & Midswing \\
3 & 010 & Midstance & Terminal swing \\
4 & 011 & Preswinal stance & Heel strike \\
5 & 100 & Toe off & Loading response \\
6 & 101 & Midswing & Midstance \\
7 & 110 & Terminal swing & Terminal stance \\
8 & 111 & Heel strike & Preswing \\
\hline
\end{tabular}

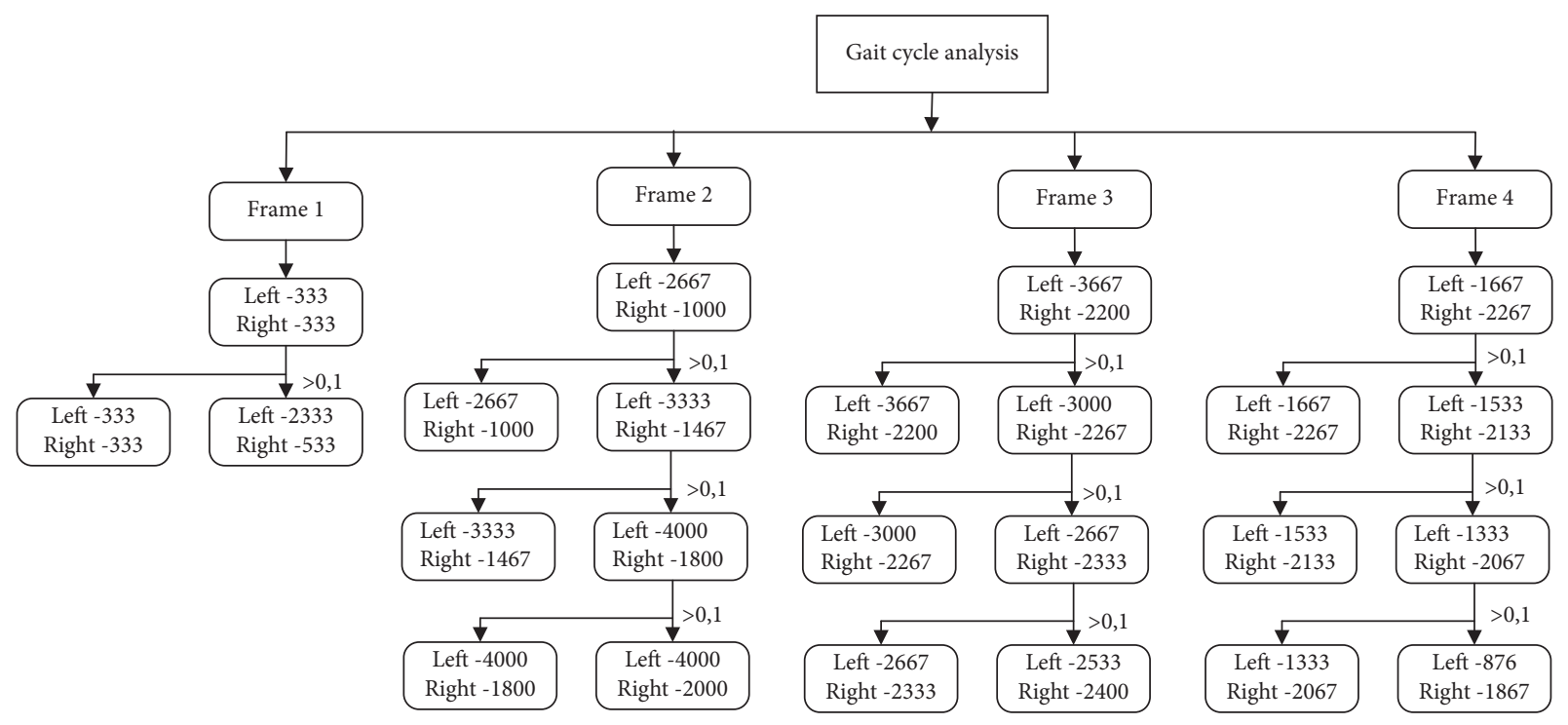

Figure 6: Decision tree scheme for the generation of assistive torque for the left hip. 


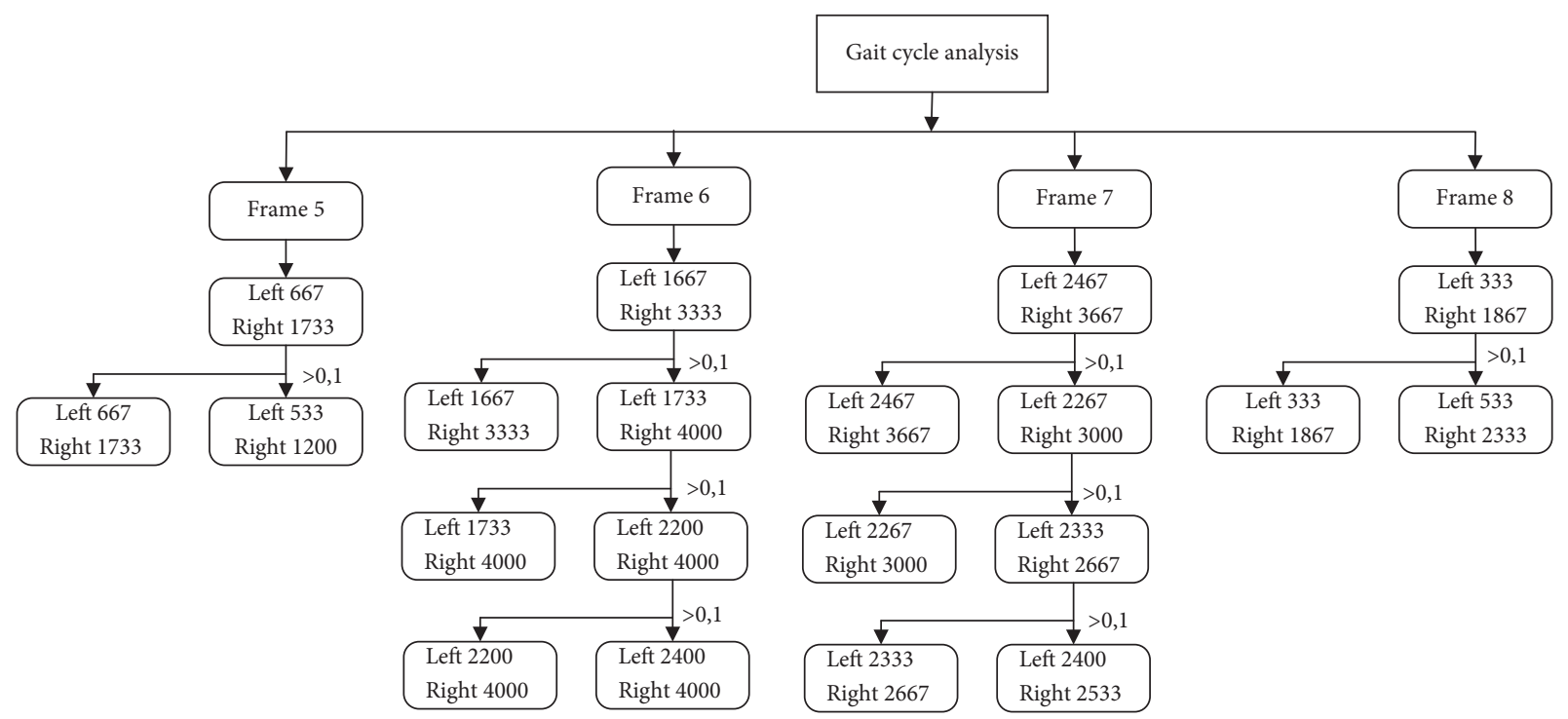

Figure 7: Decision tree scheme for the generation of assistive torque for the right hip.

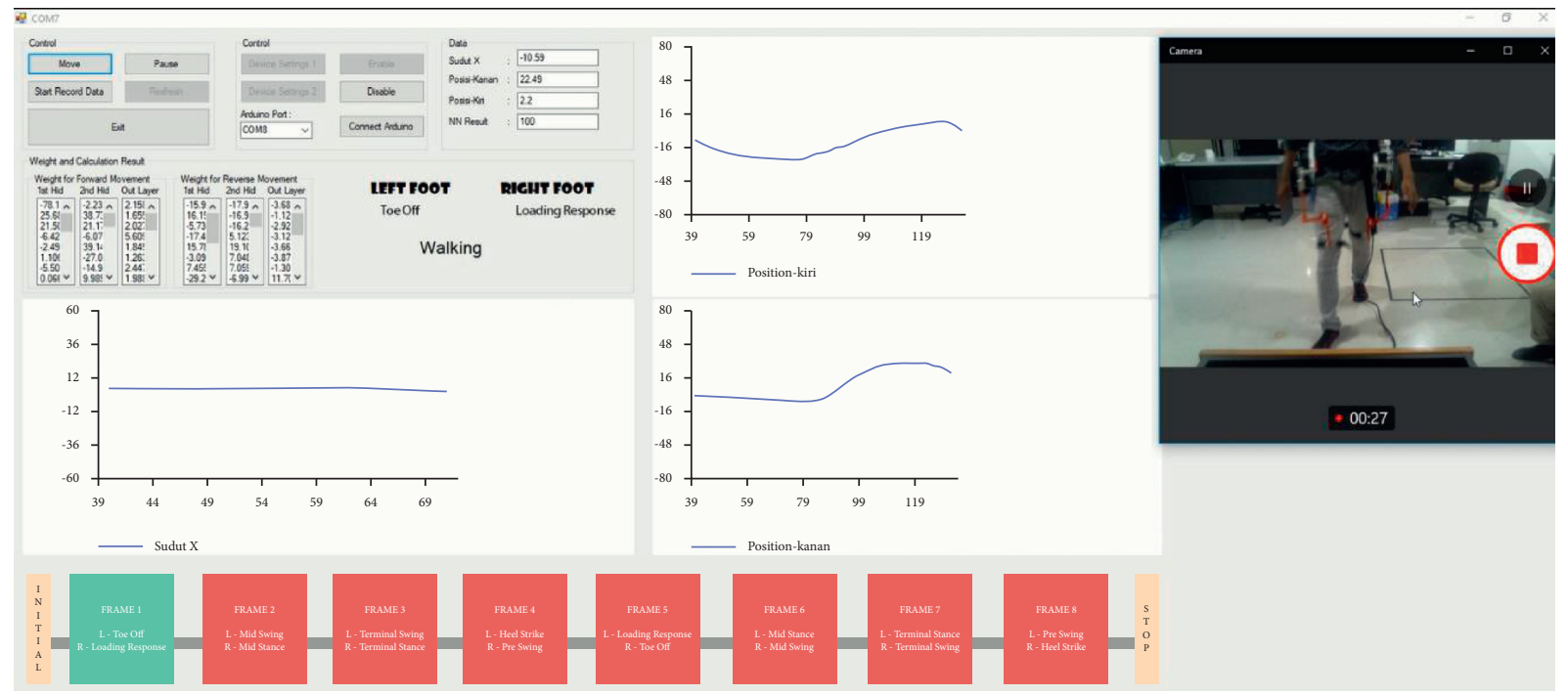

(a)

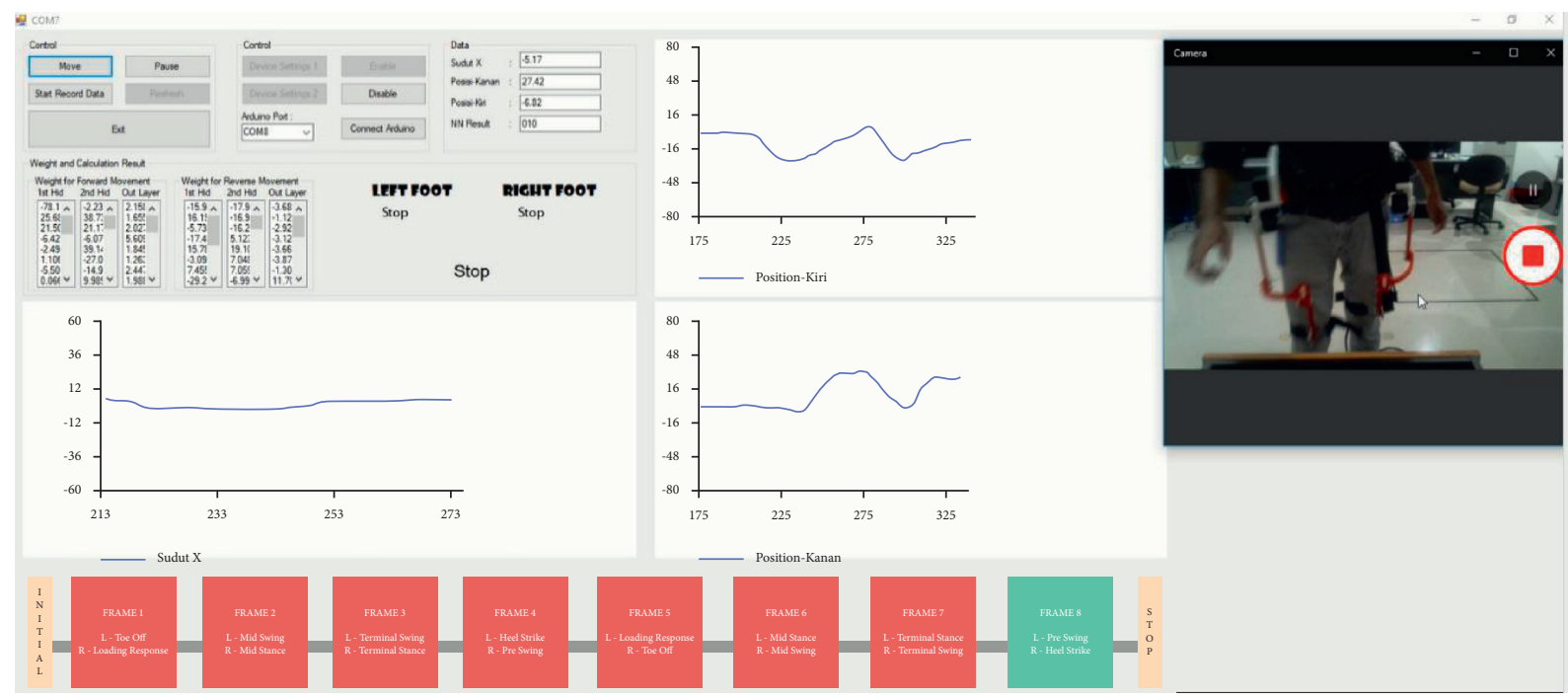

(b)

FIGURE 8: Detection of walking intention. (a) When the user is walking and (b) when the user has stopped. 
TABLE 3: Results from the proposed method of identifying walking intention.

\begin{tabular}{|c|c|c|c|c|c|c|c|c|}
\hline No. & Left angle $\left({ }^{\circ}\right)$ & Right angle $\left({ }^{\circ}\right)$ & Chest angle $\left({ }^{\circ}\right)$ & Forward/reverse/initial & Gait cycle & Left phase & Right phase & Frame \\
\hline 1 & 0.49 & 0.49 & 0.53 & 2 & 101 & Initial & Initial & 0 \\
\hline 2 & 0.49 & 0.49 & 0.53 & 2 & 101 & Initial & Initial & 0 \\
\hline 3 & 0.47 & 0.49 & 0.53 & 1 & 100 & Toe off & Loading response & 1 \\
\hline 4 & 0.44 & 0.49 & 0.52 & 1 & 100 & Toe off & Loading response & 1 \\
\hline 5 & 0.39 & 0.5 & 0.48 & 1 & 101 & Midswing & Midstance & 2 \\
\hline 6 & 0.36 & 0.5 & 0.43 & 1 & 101 & Midswing & Midstance & 2 \\
\hline 7 & 0.33 & 0.5 & 0.4 & 1 & 101 & Midswing & Midstance & 2 \\
\hline 8 & 0.3 & 0.51 & 0.38 & 1 & 101 & Midswing & Midstance & 2 \\
\hline 9 & 0.28 & 0.52 & 0.36 & 1 & 110 & Terminal swing & Terminal stance & 3 \\
\hline 10 & 0.25 & 0.53 & 0.33 & 1 & 110 & Terminal swing & Terminal stance & 3 \\
\hline 11 & 0.23 & 0.54 & 0.28 & 1 & 111 & Heel strike & Preswing & 4 \\
\hline 12 & 0.23 & 0.55 & 0.24 & 1 & 111 & Heel strike & Preswing & 4 \\
\hline 13 & 0.24 & 0.56 & 0.23 & 1 & 111 & Heel strike & Preswing & 4 \\
\hline 14 & 0.25 & 0.57 & 0.22 & 1 & 000 & Loading response & Toe off & 5 \\
\hline 15 & 0.28 & 0.58 & 0.21 & 1 & 000 & Loading response & Toe off & 5 \\
\hline 16 & 0.3 & 0.56 & 0.24 & 0 & 000 & Loading response & Toe off & 5 \\
\hline 17 & 0.33 & 0.49 & 0.28 & 0 & 000 & Loading response & Toe off & 5 \\
\hline 18 & 0.36 & 0.42 & 0.45 & 0 & 001 & Midstance & Midswing & 6 \\
\hline 19 & 0.39 & 0.37 & 0.56 & 0 & 001 & Midstance & Midswing & 6 \\
\hline 20 & 0.42 & 0.32 & 0.58 & 0 & 001 & Midstance & Midswing & 6 \\
\hline 21 & 0.45 & 0.27 & 0.59 & 0 & 001 & Midstance & Midswing & 6 \\
\hline 22 & 0.47 & 0.24 & 0.61 & 0 & 010 & Terminal stance & Terminal swing & 7 \\
\hline 23 & 0.5 & 0.22 & 0.64 & 0 & 010 & Terminal stance & Terminal swing & 7 \\
\hline 24 & 0.52 & 0.23 & 0.65 & 0 & 010 & Terminal stance & Terminal swing & 7 \\
\hline 25 & 0.55 & 0.25 & 0.63 & 0 & 011 & Preswing & Heel strike & 8 \\
\hline 26 & 0.58 & 0.25 & 0.61 & 0 & 011 & Preswing & Heel strike & 8 \\
\hline 27 & 0.59 & 0.25 & 0.61 & 0 & 011 & Preswing & Heel strike & 8 \\
\hline 28 & 0.57 & 0.26 & 0.59 & 0 & 011 & Preswing & Heel strike & 8 \\
\hline 29 & 0.52 & 0.31 & 0.47 & 1 & 100 & Toe off & Loading response & 1 \\
\hline 30 & 0.45 & 0.34 & 0.34 & 1 & 100 & Toe off & Loading response & 1 \\
\hline 31 & 0.4 & 0.39 & 0.24 & 1 & 110 & Toe off & Loading response & 1 \\
\hline 32 & 0.34 & 0.43 & 0.26 & 1 & 101 & Midswing & Midstance & 2 \\
\hline 33 & 0.31 & 0.47 & 0.25 & 1 & 111 & Midswing & Midstance & 2 \\
\hline 34 & 0.29 & 0.51 & 0.23 & 1 & 101 & Midswing & Midstance & 2 \\
\hline 35 & 0.28 & 0.54 & 0.22 & 1 & 110 & Terminal swing & Terminal stance & 3 \\
\hline 36 & 0.28 & 0.56 & 0.21 & 1 & 111 & Heel strike & Preswing & 4 \\
\hline 37 & 0.28 & 0.58 & 0.2 & 1 & 111 & Heel strike & Preswing & 4 \\
\hline 38 & 0.28 & 0.59 & 0.2 & 1 & 000 & Loading response & Toe off & 5 \\
\hline 39 & 0.29 & 0.59 & 0.19 & 1 & 111 & Loading response & Toe off & 5 \\
\hline 40 & 0.3 & 0.54 & 0.25 & 1 & 111 & Loading response & Toe off & 5 \\
\hline 41 & 0.35 & 0.46 & 0.32 & 0 & 000 & Loading response & Toe off & 5 \\
\hline 42 & 0.38 & 0.4 & 0.48 & 0 & 001 & Midstance & Midswing & 6 \\
\hline 43 & 0.42 & 0.34 & 0.49 & 0 & 001 & Midstance & Midswing & 6 \\
\hline 44 & 0.46 & 0.29 & 0.48 & 0 & 001 & Midstance & Midswing & 6 \\
\hline 45 & 0.5 & 0.26 & 0.5 & 0 & 001 & Midstance & Midswing & 6 \\
\hline 46 & 0.53 & 0.25 & 0.52 & 0 & 010 & Terminal stance & Terminal swing & 7 \\
\hline 47 & 0.55 & 0.25 & 0.55 & 0 & 11 & Preswing & Heel strike & 8 \\
\hline 48 & 0.58 & 0.26 & 0.58 & 0 & 11 & Preswing & Heel strike & 8 \\
\hline 49 & 0.6 & 0.26 & 0.6 & 0 & 11 & Preswing & Heel strike & 8 \\
\hline 50 & 0.6 & 0.26 & 0.6 & 0 & 11 & Preswing & Heel strike & 8 \\
\hline 51 & 0.57 & 0.29 & 0.55 & 0 & 11 & Preswing & Heel strike & 8 \\
\hline 52 & 0.5 & 0.33 & 0.46 & 1 & 100 & Toe off & Loading response & 1 \\
\hline 53 & 0.44 & 0.36 & 0.35 & 1 & 100 & Toe off & Loading response & 1 \\
\hline 54 & 0.39 & 0.4 & 0.36 & 1 & 100 & Toe off & Loading response & 1 \\
\hline 55 & 0.34 & 0.44 & 0.36 & 1 & 100 & Toe off & Loading response & 1 \\
\hline 56 & 0.31 & 0.48 & 0.34 & 1 & 101 & Midswing & Midstance & 2 \\
\hline 57 & 0.29 & 0.52 & 0.34 & 1 & 101 & Midswing & Midstance & 2 \\
\hline 58 & 0.29 & 0.54 & 0.33 & 1 & 110 & Terminal swing & Terminal stance & 3 \\
\hline 59 & 0.29 & 0.56 & 0.31 & 1 & 110 & Terminal swing & Terminal stance & 3 \\
\hline 60 & 0.28 & 0.58 & 0.27 & 1 & 111 & Heel strike & Preswing & 4 \\
\hline
\end{tabular}


TABLE 3: Continued.

\begin{tabular}{|c|c|c|c|c|c|c|c|c|}
\hline No. & Left angle $\left({ }^{\circ}\right)$ & Right angle $\left({ }^{\circ}\right)$ & Chest angle $\left({ }^{\circ}\right)$ & Forward/reverse/initial & Gait cycle & Left phase & Right phase & Frame \\
\hline 61 & 0.27 & 0.59 & 0.25 & 1 & 111 & Heel strike & Preswing & 4 \\
\hline 62 & 0.3 & 0.59 & 0.25 & 1 & 111 & Heel strike & Preswing & 4 \\
\hline 63 & 0.31 & 0.53 & 0.32 & 0 & 000 & Loading response & Toe off & 5 \\
\hline 64 & 0.36 & 0.46 & 0.38 & 0 & 000 & Loading response & Toe off & 5 \\
\hline 65 & 0.39 & 0.41 & 0.49 & 0 & 001 & Midstance & Midswing & 6 \\
\hline 66 & 0.45 & 0.34 & 0.51 & 0 & 001 & Midstance & Midswing & 6 \\
\hline 67 & 0.48 & 0.3 & 0.5 & 0 & 001 & Midstance & Midswing & 6 \\
\hline 68 & 0.51 & 0.28 & 0.56 & 0 & 001 & Midstance & Midswing & 6 \\
\hline 69 & 0.53 & 0.28 & 0.61 & 0 & 010 & Terminal stance & Terminal swing & 7 \\
\hline 70 & 0.55 & 0.28 & 0.65 & 0 & 011 & Preswing & Heel strike & 8 \\
\hline 71 & 0.56 & 0.29 & 0.67 & 0 & 011 & Preswing & Heel strike & 8 \\
\hline 72 & 0.56 & 0.28 & 0.66 & 0 & 011 & Preswing & Heel strike & 8 \\
\hline 73 & 0.55 & 0.29 & 0.66 & 0 & 011 & Preswing & Heel strike & 8 \\
\hline 74 & 0.51 & 0.31 & 0.58 & 1 & 100 & Toe off & Loading response & 1 \\
\hline 75 & 0.44 & 0.33 & 0.56 & 1 & 100 & Toe off & Loading response & 1 \\
\hline 76 & 0.4 & 0.36 & 0.5 & 1 & 100 & Toe off & Loading response & 1 \\
\hline 77 & 0.38 & 0.39 & 0.51 & 1 & 101 & Midswing & Midstance & 2 \\
\hline 78 & 0.36 & 0.41 & 0.52 & 1 & 101 & Midswing & Midstance & 2 \\
\hline 79 & 0.37 & 0.43 & 0.5 & 1 & 111 & Midswing & Midstance & 2 \\
\hline
\end{tabular}

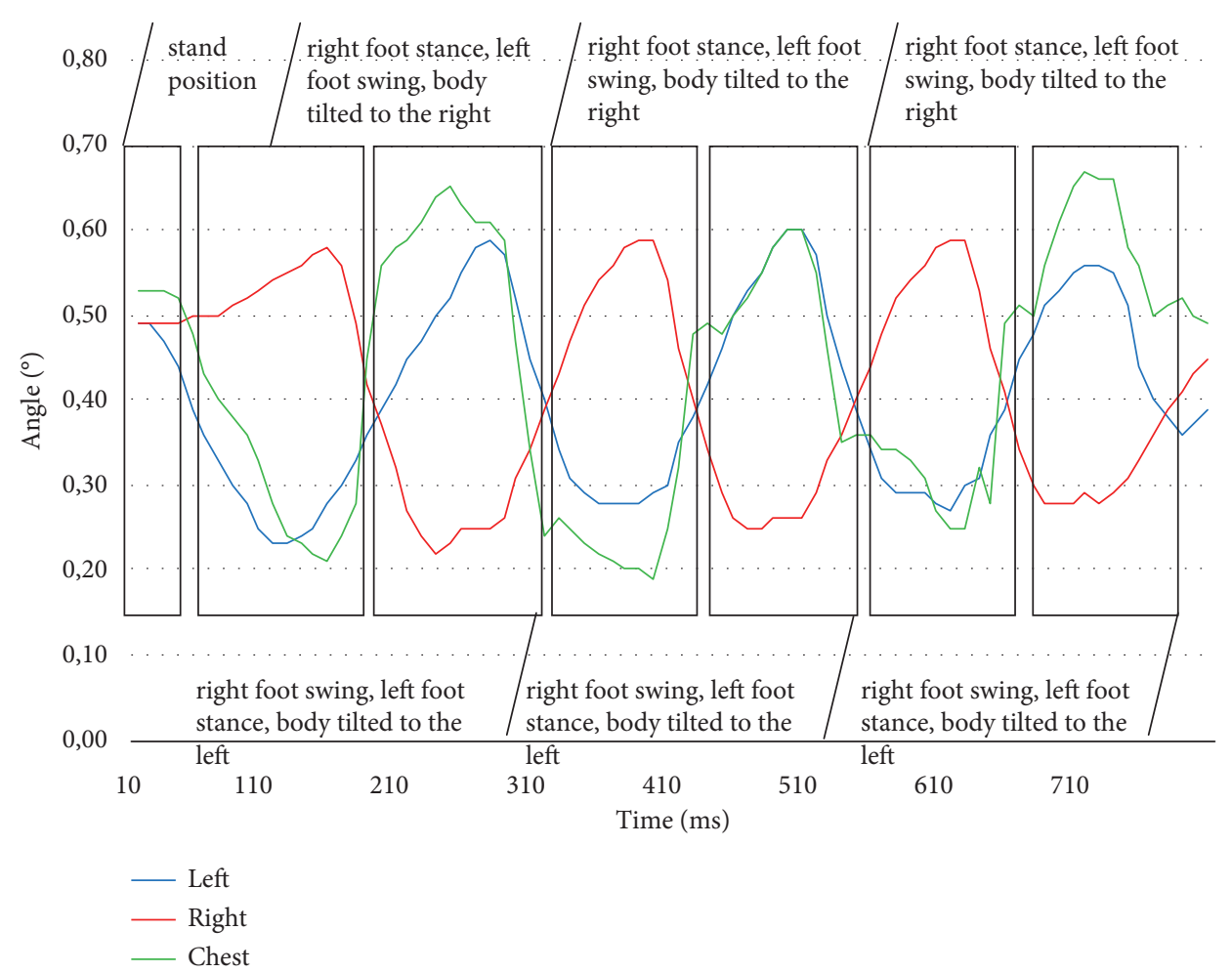

Figure 9: Complete signal representing a normal human walking cycle.

very high. Our scheme can be compared to the work in $[7,8]$, in which a complete lower limb exoskeleton was developed for the hip, knee, and ankle joints. In [7], the system was able to provide around $30 \mathrm{~N}$ of assistive torque for the hip and $5 \mathrm{~N}$ for the knee joint when a user was walking at normal speed, while in [8], a complete lower limb exoskeleton was validated by pushing the body to the wall and was able to provide an assistive torque of around $24.8 \mathrm{Nm}$ for the ankle, $17.8 \mathrm{Nm}$ for the knee joint, and $9.8 \mathrm{Nm}$ for the hip joint. 


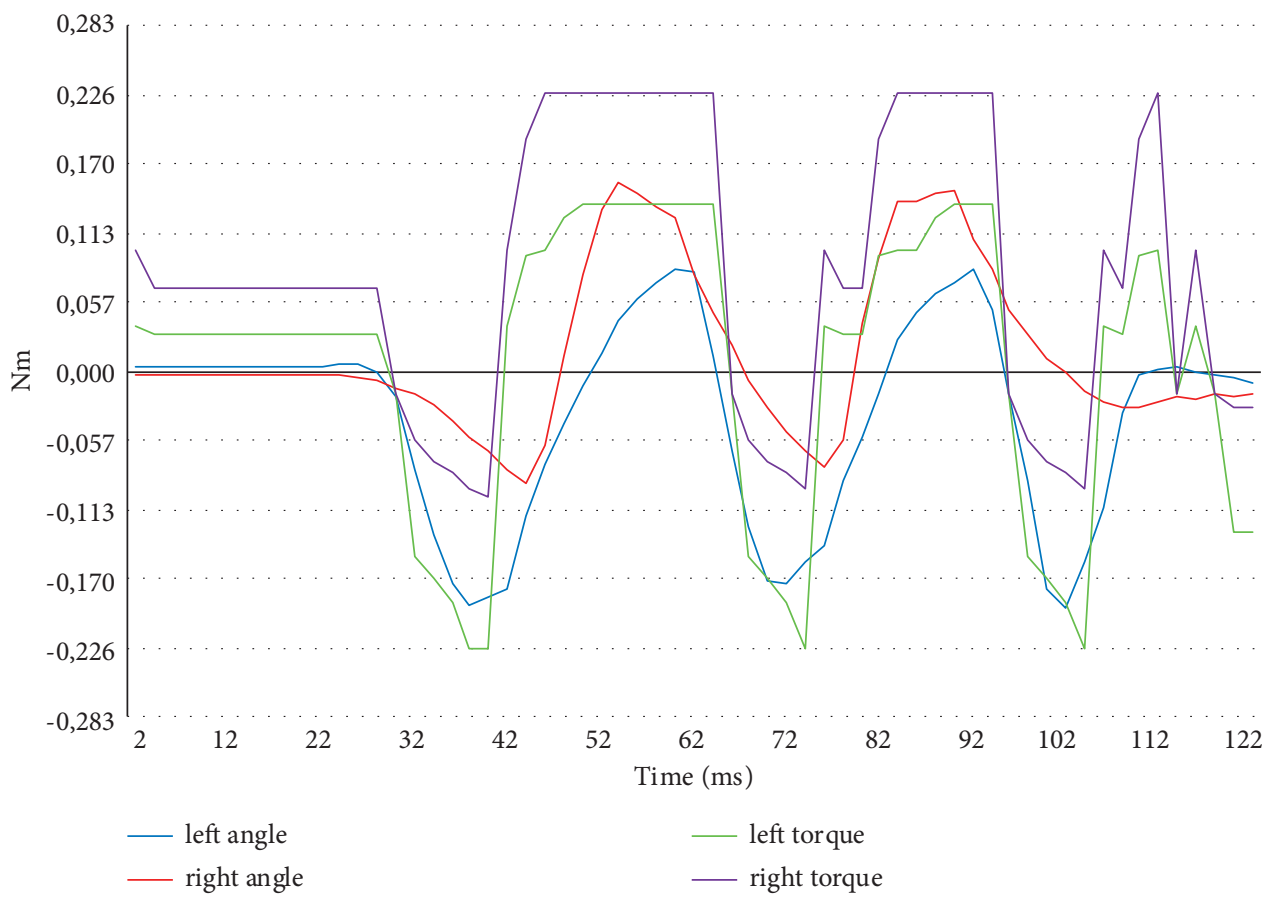

Figure 10: Assistive torque generated for a normal walking process.

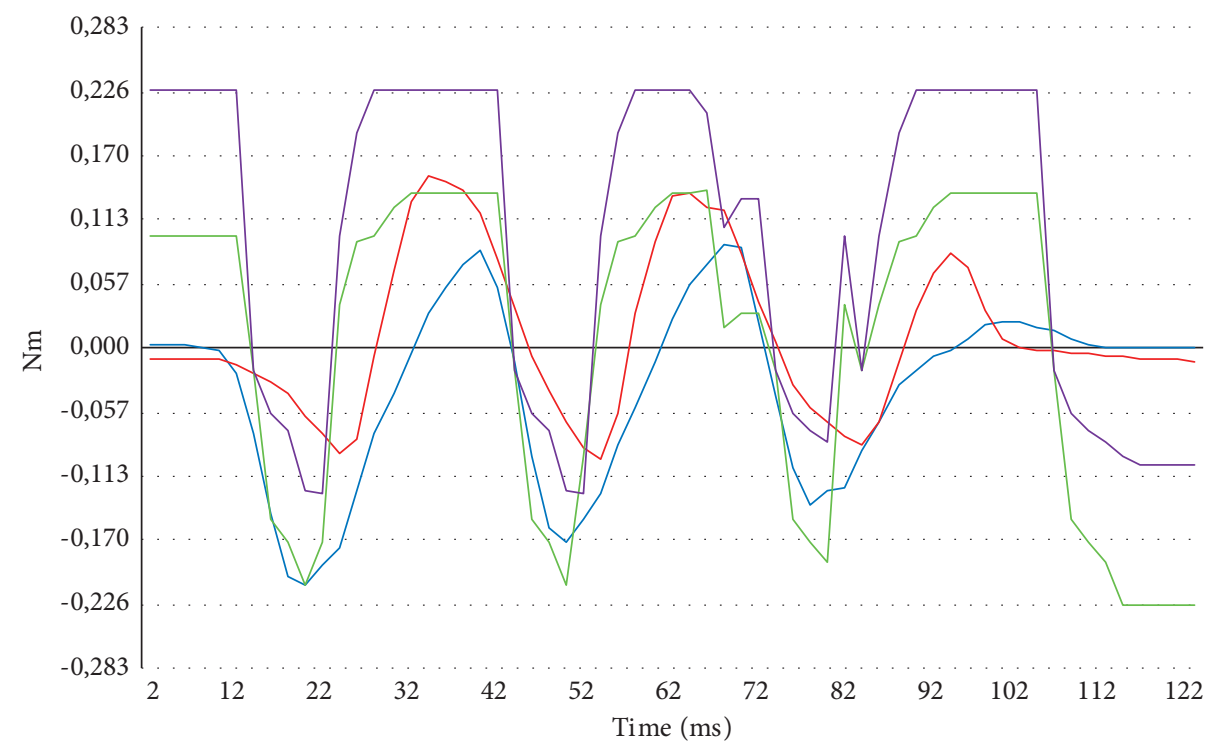

$$
\begin{array}{ll}
\text { — left angle } & \text { - left torque } \\
\text { right angle } & \text { right torque }
\end{array}
$$

FIGURE 11: Assistive torque generated for the left and right hip joints with a wider swing step. 


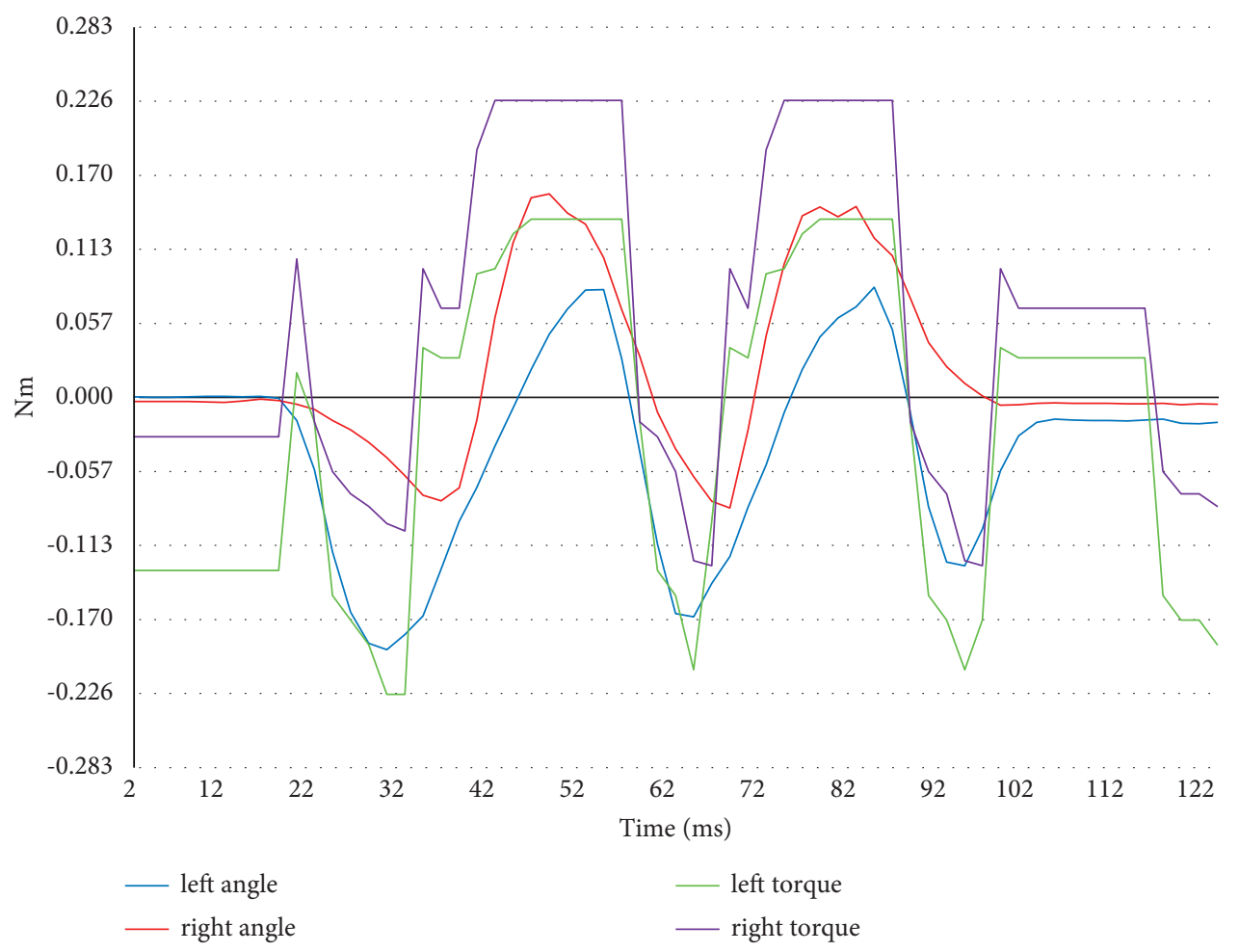

Figure 12: Assistive torque generated for the left and right hip joints for a faster walking pace.

\section{Conclusion}

This paper has proposed a walking gait classification system to detect a human being's intention to walk based on an IMU sensor and an encoder. Walking classification was achieved using an $\mathrm{NN}$ module and was implemented in a real-time application, and a decision tree method was used to generate the assistive torque. Although we carried out only simple experiments, our system was able to correctly identify the intention to walk based on the initial step. Assistive torque was generated based on the results for walking intention. Our experiments showed that the user needed more torque for the right foot than for the left, and this caused the appearance of a flat signal on the graph as the torque exceeded the maximum limit of the motors. Our mechanical design did not include a conversion gear, in order to avoid the generation of a tremendous torque by the motor. A huge torque would force the user to move their legs, and it is very risky in terms of user safety. The method used to generate assistive torque is still under development, and in future work, we will consider implementing a conversion gear that is suitable for the user's needs without compromising comfort.

\section{Data Availability}

The data used to support the findings of this study are available from the corresponding author upon request.

\section{Conflicts of Interest}

The authors declare that they have no conflicts of interest.

\section{Acknowledgments}

This work was fully supported by the Ministry of Research, Technology, and Higher Education of the Republic of Indonesia.

\section{References}

[1] D. S. Pamungkas, W. Caesarendra, H. Soebakti, R. Analia, and S. Susanto, "Overview: types of lower limb exoskeletons," Electronics, vol. 8, no. 11, p. 1283, 2019.

[2] H. Kazerooni, J. Racine, L. Huang, and R. Steger, "On the control of the Berkeley lower extremity exoskeleton (BLEEX)," in Proceedings of the 2005 IEEE International Conference on Robotics and Automation, pp. 4353-4360, Barcelona, Spain, 2005.

[3] Lockheed Martin: https://www.lockheedmartin.com/en-us/ products/exoskeleton-technologies/military.html.

[4] Rewalk by ARGO Medical Technologies, Inc. http://www. rewalk.com.

[5] A. Tsukahara, Y. Hasegawa, K. Eguchi, and Y. Sankai, "Restoration of gait for spinal cord injury patients using HAL with intention estimator for preferable swing speed," IEEE Transactions on Neural Systems and Rehabilitation Engineering, vol. 23, no. 2, pp. 308-318, 2015.

[6] K. Song, Y. Chien, and D. Susanto, "Development of an assistive torque generation system for a lower limb exoskeleton," in Proceedings of the 2016 International Conference on System Science and Engineering (ICSSE), pp. 1-4, Puli, Taiwan, July 2016.

[7] R. A. Susanto and K. Song, "Design of assistive torque for a lower limb exoskeleton based on motion prediction," in Proceedings of the 55th Annual Conference of the Society of Instrument and Control Engineers of Japan (SICE), pp. 172177, Tsukuba, Japan, March 2016. 
[8] L. Saccares, A. Brygo, I. Sarakoglou, and N. G. Tsagarakis, “A novel human effort estimation method for knee assistive exoskeletons," in Proceedings of the 2017 International Conference on Rehabilitation Robotics (ICORR), pp. 1266-1272, London, UK, October 2017.

[9] L. Saccares, I. Sarakoglou, and N. G. Tsagarakis, "A novel joint torque estimation method and sensory system for assistive lower limb exoskeletons," in Proceedings of the 2018 IEEE/RSJ International Conference on Intelligent Robots and Systems (IROS), pp. 1-9, Madrid, Spain, October 2018.

[10] V. M. Akhil, J. Varghese, P. K. Rajendrakumar, and K. S. Sivanandan, "Torque required at the knee joint of a robotic assistive device for its thigh to follow the parabolic trajectory generated by its hip joint during sit-to-stand posture," in Proceedings of the 2017 International Conference on Innovations in Electrical, Electronics, Instrumentation and Media Technology (ICEEIMT), pp. 7-10, Coimbatore, India, May 2017.

[11] S. M. Tahamipour-Z, S. K. Hosseini Sani, A. Akbarzadeh, and I. Kardan, "An assistive strategy for compliantly actuated exoskeletons using non-linear model predictive control method," in Proceedings of the Electrical Engineering (ICEE), Iranian Conference on, pp. 982-987, Mashhad, Iran, July 2018.

[12] T. Xue, Z. Wang, T. Zhang, M. Zhang, and Z. Li, "The control system for flexible hip assistive exoskeleton," in Proceedings of the 2018 IEEE International Conference on Robotics and Biomimetics (ROBIO), pp. 697-702, Kuala Lumpur, Malaysia, July 2018.

[13] A. Thongsook, T. Nunthawarasilp, P. Kraypet, J. Lim, and N. Ruangpayoongsak, "C4.5 decision tree against neural network on gait phase recognition for lower limp exoskeleton," in Proceedings of the 2019 First International Symposium on Instrumentation, Control, Artificial Intelligence, and Robotics (ICA-SYMP), pp. 69-72, Bangkok, Thailand, July 2019.

[14] W. Wang and L. Fu, "Mirror therapy with an exoskeleton upper-limb robot based on imu measurement system," in Proceedings of the 2011 IEEE International Symposium on Medical Measurements and Applications, pp. 370-375, Bari, Italy, March 2011.

[15] M. G. B. Atia and O. Salah, "Fuzzy logic with load compensation for upper limb exoskeleton control based on IMU data fusion," in Proceedings of the 2018 IEEE International Conference on Robotics and Biomimetics (ROBIO), pp. 2147-2152, Kuala Lumpur, Malaysia, July 2018.

[16] F. Patane, S. Rossi, F. Del Sette, J. Taborri, and P. Cappa, "WAKE-Up exoskeleton to assist children with cerebral palsy: design and preliminary evaluation in level walking," IEEE Transactions on Neural Systems and Rehabilitation Engineering: A Publication of the IEEE Engineering in Medicine and Biology Society, vol. 25, no. 7, pp. 906-916, 2017.

[17] W. Pirker and R. Katzenschlager, Gait Disorders in Adults and the Elderly, SpringerLink, Berlin, Germany, 2016.

[18] Y. Y. Song and Y. Lu, "Decision tree methods: applications for classification and prediction," Shanghai Archives of Psychiatry, vol. 27, no. 2, pp. 130-135, 2015.

[19] D. Miranda-Linares, G. Alrezage, and M. O. Tokhi, "Control of lower limb exoskeleton for elderly assistance on basic mobility tasks," in Proceedings of the 19th International Conference on System Theory, Control and Computing (ICSTCC), pp. 441-446, Romania, Balkans, May 2015. 